\title{
Heurística da Ancoragem na Decisão de Especialistas: Resultados Sob Teste de Manipulação
}

\author{
Anchoring Heuristic in Experts Decision: Results Under the Manipulation Test
}

Paula Borges Tronco ${ }^{1}$

(iD) https://orcid.org/0000-0003-3600-4161

Mauri Leodir Löbler ${ }^{1}$

(D) https://orcid.org/0000-0002-0244-6351

Leticia Gomes dos Santos ${ }^{1}$

(iD) https://orcid.org/0000-0002-6173-2606

Juliana Mayumi Nishi ${ }^{1}$

(iD) https://orcid.org/0000-0003-1826-5763

Universidade Federal de Santa Maria, Programa de Pós-Graduação em Administração, Santa Maria, ES, Brasil ${ }^{1}$

Artigo recebido em 02.07.2018. Última versão recebida em 15.02.2019. Aprovado em 19.02.2019. 


\title{
Resumo
}

Embora as decisões auxiliadas pela tecnologia tenham avançado nos últimos anos, o processo de decidir ainda é uma atividade essencialmente humana. Variáveis pessoais do decisor ou de contexto interferem nesse processo, com destaque para a variável conhecimento, tendo, por isso, ocupado um lugar cada vez mais relevante na literatura, ao se buscar verificar sua interveniência. Os vieses cognitivos, tais como a ancoragem, constituem, desse modo, o foco de diversos estudos que buscam averiguar sua influência, bem como o nível de conhecimento do decisor (seja especialista ou não especialista) sobre o assunto objeto da decisão. Assim, surge o problema desta pesquisa consiste em saber se o efeito da heurística da ancoragem é afetado pela manipulação do conhecimento dos decisores. Com o intuito de responder a essa pergunta, foi realizado um quase-experimento com 324 sujeitos decisores, divididos em Grupos de Calibragem e Grupos Experimentais, utilizando-se o Modelo de Jacowitz e Kahneman (1995). Como resultado principal, proveniente do teste de manipulação (Cozby, 2006) da variável de controle (o conhecimento), observa-se a não verificação de ancoragem em indivíduos especialistas submetidos a uma tarefa decisória relacionada ao seu campo de conhecimento, contrariando os achados de Northcraft e Neale (1987) e Dorow (2009).

Palavras-chave: ancoragem; vieses; nível de conhecimento; especialistas.

\begin{abstract}
Although technology-assisted decisions have advanced in recent years, the decision-making process is still essentially human. Personal decision or context variables are explored throughout the process and the variable knowledge appears as important for literature, when looking to verify its intervention. Cognitive biases, among them anchoring, are the focus of several studies on their influence, as well as the level of knowledge of the decisionmaker on the subject matter of the decision, classified as specialists and novices. Thus, the research problem arises: is the effect of Anchoring Heuristics affected by the manipulation of decision makers' knowledge? In order to answer this question, a quasi-experiment was performed with 324 decision subjects, divided into Calibration Groups and Experimental Groups, using the Jacowitz and Kahneman Model (1995). The study brings as its main results from the Manipulation Test (Cozby, 2006) of the knowledge variable the lack of anchoring effect in experts decision related to their field of knowledge, these results going against Northcraft e Neale (1987) e Dorow (2009).
\end{abstract}

Keywords: anchoring; biases; levels of knowledge; specialists.

JEL Code: C91, D9, D81. 


\section{Introdução}

As atividades humanas estão associadas a um processo de escolha e julgamento, o que implica um processo de tomada de decisão. Tomar decisões é um ato complexo que envolve a definição do problema, a busca de informações, o estabelecimento de critérios para avaliação, assim como outras fases voltadas a encontrar a melhor opção dentre as possibilidades existentes (Löbler, Reis, Nishi, \& Tagliapietra, 2015). A decisão, dessa forma, pode ser observada em suas fases e também classificada.

Por outro lado, as pessoas possuem a tendência de procurar por pontos de referência quando as informações são insuficientes ou ambíguas para a tomada de decisão (Jung \& Young, 2012). Há fatores no processo decisório que podem levar o decisor a buscar e demonstrar estratégias diferentes na decisão, como comentam Jung e Young (2012), pois os cenários de decisão não são simples ou diretos. A investigação na área da Economia Comportamental produziu evidências sobre a complexidade do processo decisório, seus vieses e irracionalidades, que pedem a reavaliação do decisor racional proposto inicialmente pela Economia, demonstrando o frequente afastamento existente entre as decisões reais e a descrição das decisões previstas pelos modelos formais de raciocínio (Magalhães, 2013).

Para que o processo decisório seja simplificado, Simon (1955) postula a necessidade de uma racionalidade limitada, de forma que somente os fatores que estão ligados, casual e temporalmente, à decisão sejam levados em consideração. $\mathrm{O}$ autor também afirma que os tomadores de decisão têm limitações em suas habilidades no processamento de informações e, consequentemente, não podem ser perfeitamente racionais.

Nesse contexto, no início dos anos 70, estudos de dois psicólogos israelenses, Amos Tversky e Daniel Kahneman (1974), revolucionaram a pesquisa acadêmica referente ao julgamento e à tomada de decisão humana. Os autores definiram que julgamentos em situações de incerteza são frequentemente baseados em um número limitado de heurísticas simplificadoras, e não no processamento de um algoritmo mais formal e extenso. Assim, em 1974, por meio do artigo Judgment under uncertainty: heuristics and biases, os psicólogos identificaram as heurísticas e os vieses que afetam o tomador de decisão quando se depara com a incerteza: heurística da disponibilidade; heurística da representatividade e heurística da ancoragem e do ajuste. Sob essa ótica, Bazerman (2004) afirma que ocorre um viés cognitivo quando os indivíduos empregam uma heurística de maneira inadequada durante seu processo decisório.

Chao (2011) entende que os decisores tendem a se basear em heurísticas sob condições de incerteza, buscando, dessa forma, reduzir a complexidade do problema. As heurísticas são, então, processos que reduzem tarefas complexas de avaliar, com o intuito de simplificar a operação de julgamento (Costa, 2017).

A ancoragem, terceira heurística identificada por Tversky e Kahneman, que consiste no objeto de estudo deste trabalho, baseia-se na ideia de que os tomadores de decisão, no desenvolvimento de suas estimativas finais, ajustam o valor à âncora considerada, mas tendem a ajustar insuficientemente a partir desse ponto (Luppe, 2006).

Para Mochon e Frederick (2013), ancoragem é o termo aplicado a situações nas quais os julgamentos e as escolhas numéricas são realizadas em direção a medidas previamente estabelecidas. Nessa perspectiva, Jung e Young (2012) afirmam que a ancoragem é um fenômeno robusto, o qual já foi reportado em inúmeros contextos diferentes, tais como apostas, estimativas de probabilidades e fatores egocêntricos. Além disso, tal fenômeno pode ser percebido não só no contexto de laboratório, mas também entre profissionais experientes (Loschelder, Friese, \& Trötschel, 2017).

Alguns estudos demonstraram a consistência da heurística da ancoragem. Northcraft e Neale (1987) mostraram, por exemplo, que corretores de imóveis avaliaram propriedades baseados em um número inicial que lhes era dado, fornecendo avaliações diferentes para a mesma propriedade conforme o número indicado. Em outro estudo, Mussweiler e Strack (2000) utilizaram vendedores de automóveis 
para estimar o preço de um automóvel que desejavam vender. Os autores apresentaram um valor de âncora alto e um baixo aos respondentes e esses, mesmo sendo especialistas, foram influenciados pelos valores das âncoras. Assim sendo, os autores concluíram que tanto pessoas leigas quanto especialistas no assunto estão propensas ao efeito da ancoragem. Neste estudo, com a intenção de contribuir com o argumento em questão, a pressuposição de Northcraft e Neale (1987) e de Mussweiler e Strack (2000) é testada.

No entanto, Thorsteinson, Breier, Atwell, Hamilton e Privette (2008) e Welsh, Delfabbro, Burns e Begg (2014) afirmam que os efeitos da ancoragem podem ser reduzidos quando sujeitos decisores têm mais conhecimento acerca dos problemas em questão. Nessa perspectiva, Dorow (2009) afirma que, quanto menos um indivíduo conhece um assunto, objeto ou produto, maiores são as probabilidades de ser influenciado por um valor arbitrário (âncora).

Considerando tais achados e também o estudo inicial e contraditório de Northcraft e Neale (1987), a variável conhecimento ocupa papel central nesta pesquisa, sendo uma das mais relevantes contribuições dos resultados encontrados e colaborando, por meio destes, na resolução do gap teórico.

No contexto brasileiro, também se observaram os efeitos da heurística da ancoragem. Luppe (2006) realizou um estudo tendo como objetivo examinar os efeitos da heurística da ancoragem nas estimativas numéricas sob a perspectiva da avaliação de bens de consumo. $\mathrm{O}$ autor, por meio de dois experimentos com alunos de graduação de Administração, Contábeis e Economia, demonstrou a manifestação dos efeitos da ancoragem na estimativa de quantidades incertas e de preços de diferentes produtos e serviços.

Outro estudo realizado no Brasil foi o de Dorow (2009), que desenvolveu uma pesquisa junto a corretores profissionais em investimentos imobiliários com o intuito de examinar a extensão dos efeitos causados pela heurística da ancoragem em estimativas quantitativas realizadas por esses profissionais ao efetuarem avaliações de investimentos imobiliários. O autor concluiu que os efeitos e a influência da heurística da ancoragem em investimentos imobiliários são notórios; contudo, não são extraordinariamente grandes.

Em razão do exposto, e com a intenção de contribuir com os estudos sobre a heurística da ancoragem, especialmente quanto a especialistas em mercados específicos, complementando o trabalho de Dorow (2009), este estudo vem à tona. Buscou-se, assim, identificar os efeitos da heurística da ancoragem em indivíduos especialistas em mercado imobiliário (corretores de imóveis) e indivíduos não especialistas nesse setor mediante experimento contendo duas tarefas decisórias, uma relacionada ao mercado imobiliário, e outra referente a bens de consumo (teste de manipulação).

Segundo Luppe (2006), estudos de ancoragem em tarefas de estimação frequentemente utilizam o modelo tradicional de dois estágios, de modo que os sujeitos decisores são inicialmente questionados se um valor particular (âncora) é maior ou menor que uma quantidade incerta e, então, eles estimam essa quantidade.

Este estudo, no entanto, utilizou o Modelo de Jacowitz e Kahneman (1995), o qual difere do modelo tradicional de dois estágios, pois apresenta um parâmetro para a mensuração dos efeitos da ancoragem nas tarefas de estimação e propõe um procedimento para medir a ancoragem que requer três grupos, os quais devem ser retirados de uma mesma população, denominados de grupo de calibragem (formado por especialistas) e grupos experimentais (composto de não especialistas). Esta se caracteriza como uma das principais contribuições deste trabalho: a verificação da manifestação da ancoragem quando uma variável de controle, nesse caso o conhecimento, é retirada da tarefa decisória, no procedimento descrito por Cozby (2006) como um teste de manipulação.

Assim, este estudo demonstra uma abordagem completa e inovadora, ao utilizar o Modelo de Jacowitz e Kahneman (1995), e ainda apresenta uma importante contribuição à literatura ao questionar o conhecimento dos especialistas e a possível contradição desse conhecimento frente às heurísticas, por meio do teste de manipulação, indagando se, mesmo no campo de conhecimento do especialista, prevalece o efeito da heurística da ancoragem. O objetivo geral desta pesquisa consiste, então, em 
verificar a existência da heurística da ancoragem nas estimativas de especialistas e não especialistas no mercado imobiliário, quando submetidos a tarefas experimentais relacionadas ao campo de domínio do especialista e fora do seu campo de conhecimento.

\section{Fundamentação Teórica}

\section{Processo decisório: evolução das abordagens}

O processo decisório é um componente fundamental do comportamento humano (Tversky \& Kahneman, 1974). Na concepção de Bazerman (2004), o processo de tomada de decisão deve levar em consideração três pontos importantes: os aspectos cognitivos do processo decisório; o processo mental de formar opinião ou avaliar, por meio de discernimento ou comparação; e a capacidade de julgar, ou seja, o poder e/ou a habilidade de decidir com base em evidências.

Os estudos sobre processo decisório podem abranger diferentes cenários, decisores e áreas do conhecimento. Löbler, Reis, Nishi e Tagliapietra (2015) afirmam que o amplo conjunto de áreas interessadas na compreensão do processo decisório pode ser tanto uma vantagem ao processo, disponibilizando uma vasta gama de ideias e trabalhos como fontes de conhecimento, quanto uma desvantagem, trazendo termos e conceitos ambíguos para a pesquisa. Dessa forma, é relevante a identificação clara dos conceitos, do processo e das nuances que serão tratadas especificamente.

Segundo Luppe (2006), o processo tradicional de tomada de decisões é baseado no modelo clássico de escolha racional, o qual segue uma estrutura normativa que apresenta como base primordial a Teoria da Utilidade Esperada, de Von Neumann e Morgenstern (1947). Porém, tal abordagem normativa é contestada por diversos autores, os quais afirmam que a complexidade do processo decisório não pode ser simplificada de forma puramente racional (Löbler et al., 2015; Simon, 1991).

Dentre as possíveis decisões necessárias aos indivíduos, muitas se apresentam em diferentes contextos, e muitas são decisões importantes quanto a assuntos sobre os quais o decisor não está bem informado (Rader, Soll, \& Larrick, 2015). Tais assuntos, como, por exemplo, planos de saúde, aposentadoria e novos projetos de trabalho, para os autores, têm o potencial de trazer grandes mudanças para o indivíduo, dependendo do resultado da decisão, e, por isso, recursos que auxiliam tal processo são de grande importância (Rader et al., 2015).

Ademais, muitos são os fatores que podem influenciar esse processo, como pressão do tempo, incerteza e emoções positivas ou negativas (George \& Dane, 2016), os quais, para S. Malhotra, Zhu e Reus (2015), torna os decisores propensos à utilização de heurísticas como a ancoragem.

\section{Heurísticas cognitivas}

Os indivíduos desenvolvem regras práticas ou heurísticas para reduzir as exigências do processamento de informações e para lidar com as limitadas habilidades cognitivas que possuem no processo decisório (Tversky \& Kahneman, 1974). Conforme Bazerman (2004), as heurísticas oferecem aos administradores e a outros profissionais um modo simples de lidar com um mundo complexo, usualmente produzindo julgamentos corretos ou parcialmente corretos. No entanto, a aplicação errônea da heurística em situações inadequadas pode levar a sérios erros de julgamento (Gergaud, Plantinga, \& Ringeval-Deluze, 2017), ocasionando, muitas vezes, uma distorção na maneira como os indivíduos percebem a realidade.

Nesse contexto, destaca-se o trabalho de Tversky e Kahneman (1974), em que os autores descrevem três heurísticas utilizadas no processo decisório: heurística da disponibilidade, heurística da representatividade e heurística da ancoragem e do ajuste (objeto de estudo desta pesquisa). 
Estudos como o de Abdin, Farooq, Sultana, e Farooq (2017), por exemplo, enriquecem a pesquisa no campo das heurísticas incorporando quatro importantes componentes de heurística - excesso de confiança, representatividade, ancoragem e disponibilidade, os quais são tratados independentemente para examinar seu efeito diferencial sobre anomalias e desempenho do investimento. Assim, o relacionamento do indivíduo com os processos de heurística, bem como o relacionamento das heurísticas entre si, vem sendo estudado e aprofundado.

\section{Ancoragem}

Nos últimos quarenta anos, conforme Turner e Schley (2016), a pesquisa acerca da ancoragem recebeu grande atenção devido ao seu efeito robusto e significativo e à sua ampla aplicabilidade em uma infinidade de domínios. A heurística da ancoragem e do ajustamento, de acordo com Bazerman (2004), é aquela em que se avalia a chance de ocorrência de um evento pela colocação de uma base (âncora), fazendo-se, então, um ajuste. Os indivíduos começam a realizar suas avaliações a partir de um valor inicial, que é posteriormente ajustado para fins de uma decisão final.

O valor inicial, ou ponto de partida, dessa forma, pode ser sugerido por um precedente histórico, como fizeram S. Malhotra et al. (2015) no que concerne à decisão de valores atribuídos à compra de organizações; pela maneira pela qual um problema é apresentado; ou, ainda, por uma informação aleatória. Nas palavras dos autores, os indivíduos frequentemente confiam demais nessas âncoras e raramente questionam sua validade ou adequabilidade em uma situação particular.

A ancoragem é um dos vieses comportamentais de maior aprofundamento científico (Meub \& Proeger, 2015), e não é um processo cognitivo simples. Ela consiste em uma limitação da tomada de decisão que surge em problemas presentes em um contexto de negócios reais e afeta pessoas experientes e inexperientes (Northcraft \& Neale, 1987). Ainda, para Furnham e Boo (2011), a influência do fenômeno da ancoragem é ubíquo no julgamento humano, apresentando-se, portanto, em todas as instâncias desse julgamento.

Conforme Mussweiler e Strack (2000), a ancoragem pode ser uma das influências mais notáveis em julgamento e tomada de decisão por duas razões. Em primeiro lugar, pelo fato de que demonstrações dos efeitos da ancoragem são abundantes em vários domínios nos estudos de julgamento, incluindo questões de conhecimento geral, avaliações de loterias e jogos, estimativas de risco e incerteza, avaliação de preço de imóveis, percepções da própria eficácia, avaliações do desempenho futuro, efeitos de múltiplas âncoras no julgamento individual e em grupo, avaliações de probabilidade com auditores profissionais, negociações e aplicações no mercado de consumo. Em segundo lugar, os autores argumentam que, embora o estudo da ancoragem seja de excepcional significância prática e empírica, os mecanismos cognitivos do processo de ancoragem têm sido explorados apenas recentemente.

No entanto, existem muitas possíveis situações em que âncoras potenciais precedem ou acompanham julgamentos quantitativos e não se aplicam a esse paradigma (Mochon \& Frederick, 2013), motivo pelo qual o aprofundamento do estudo do mecanismo da ancoragem se mostra relevante. $\mathrm{O}$ estudo de Loschelder, Friese e Trötschel (2017), por exemplo, ao testar tanto aqueles que disponibilizam a âncora quanto os afetados, concluiu que, quando em evento de negociação, os negociadores não devem hesitar em ser altamente precisos, pois a âncora se estabelece de forma precisa e já no início do processo.

Para Turner e Schley (2016), o efeito da ancoragem apresenta-se claramente em diversas situações. Por isso, a realização de novos estudos, que aprofundem o conhecimento na área testando teorias e propondo testes e experimentos, é relevante no contexto atual das teorias já apresentadas.

\section{Especialistas e não especialistas e suas diferenças no processo decisório}

A ancoragem tem sido demonstrada independentemente do conhecimento especializado dos respondentes no assunto proposto (Northcraft \& Neale, 1987). No entanto, Thorsteinson et al. (2008) 
argumentam que os efeitos da ancoragem são reduzidos quando os sujeitos decisores têm mais conhecimento acerca dos problemas em questão.

Desde a primeira demonstração dos efeitos da ancoragem, Tversky e Kahneman (1974) declararam que a confiança nas heurísticas e a prevalência dos vieses não são restritas a leigos. Pesquisadores experientes estão propensos aos mesmos vieses quando pensam intuitivamente.

Northcraft e Neale (1987) realizaram um experimento com estudantes e corretores imobiliários para a estimação do valor de uma casa, fornecendo todas as informações que são tipicamente importantes para a avaliação de uma residência, além de uma visita presencial, demonstrando que tanto estudantes quanto profissionais do setor imobiliário foram influenciados pelos preços disponíveis em uma lista, que consistia na âncora apresentada aos decisores.

Em outro estudo, Mussweiler e Strack (2000) utilizaram vendedores de automóveis para estimar o preço de um automóvel que desejavam vender. Os autores apresentaram um valor de âncora alto e um valor de âncora baixo aos respondentes e observaram que esses especialistas foram influenciados pelos valores indicados.

Nesse sentido, Dorow (2009) afirma que, quanto menos um indivíduo conhece um assunto, objeto ou produto, maiores são as probabilidades de ser influenciado por um valor arbitrário (âncora). Além disso, Smith, Windschitl e Bruchmann (2013) apresentam resultados robustos quanto aos decisores com maior nível de conhecimento, demonstrando a menor influência da âncora sobre eles, e quanto à diminuição do processo de ancoragem quando esses decisores recebem mais informações relevantes sobre o estudo. Considerando tais perspectivas, pesquisadores têm usado, segundo Ceschi, Costantini, Sartori, Weller e Di Fabio (in press), vários paradigmas de decisões conhecidos juntamente com desenhos focados nos sujeitos, combinando-os com medidas de diferenças individuais.

Diante do exposto e tendo em vista que diferentes autores consideram a experiência como uma característica importante na definição de indivíduos especialistas, este estudo considerou como especialista os indivíduos que possuem experiência e prática no mercado imobiliário, isto é, corretores de imóveis atuantes nesse mercado. Como não especialistas, este estudo considerou alunos de graduação, pós-graduação e servidores públicos que não possuíssem nenhuma experiência no mercado imobiliário.

\section{Aspectos Metodológicos}

\section{Caracterização da pesquisa}

Para o desenvolvimento deste estudo, foi utilizada uma pesquisa de caráter explicativo, com delineamento experimental. Campbell e Stanley (1979) afirmam que, quando não é possível atingir o mesmo grau de controle que é utilizado na pesquisa experimental propriamente dita e a randomização é inviável, deve-se empregar o delineamento denominado quase-experimental. Esses delineamentos tentam atingir um grau de controle próximo ao dos delineamentos experimentais, para inferir que tratamento teve o efeito pretendido (Cozby, 2006). Ao longo deste estudo, apenas por uma questão de fluidez do texto, será utilizado o termo experimento.

Quanto aos grupos estudados em um experimento, N. K. Malhotra (2006) sugere dois: o Grupo Experimental (GE) e o Grupo de Controle (GC). Neste estudo, tanto o GC quanto o GE foram subdivididos em mais dois grupos: GC composto por especialistas em mercado de imóveis e GC composto por não especialistas; GE constituído por especialistas em mercado imobiliário e GE formado por não especialistas. Os Grupos de Calibragem foram submetidos a duas tarefas decisórias sem a influência de âncoras. Já os Grupos Experimentais foram submetidos a âncoras baixas e âncoras altas no momento da tomada de decisão. 


\section{Modelo de pesquisa}

\section{Desenho de pesquisa}

O desenho de pesquisa na fase de calibragem é apresentado na Figura 1, com o intuito de proporcionar uma melhor compreensão das etapas seguidas neste estudo.

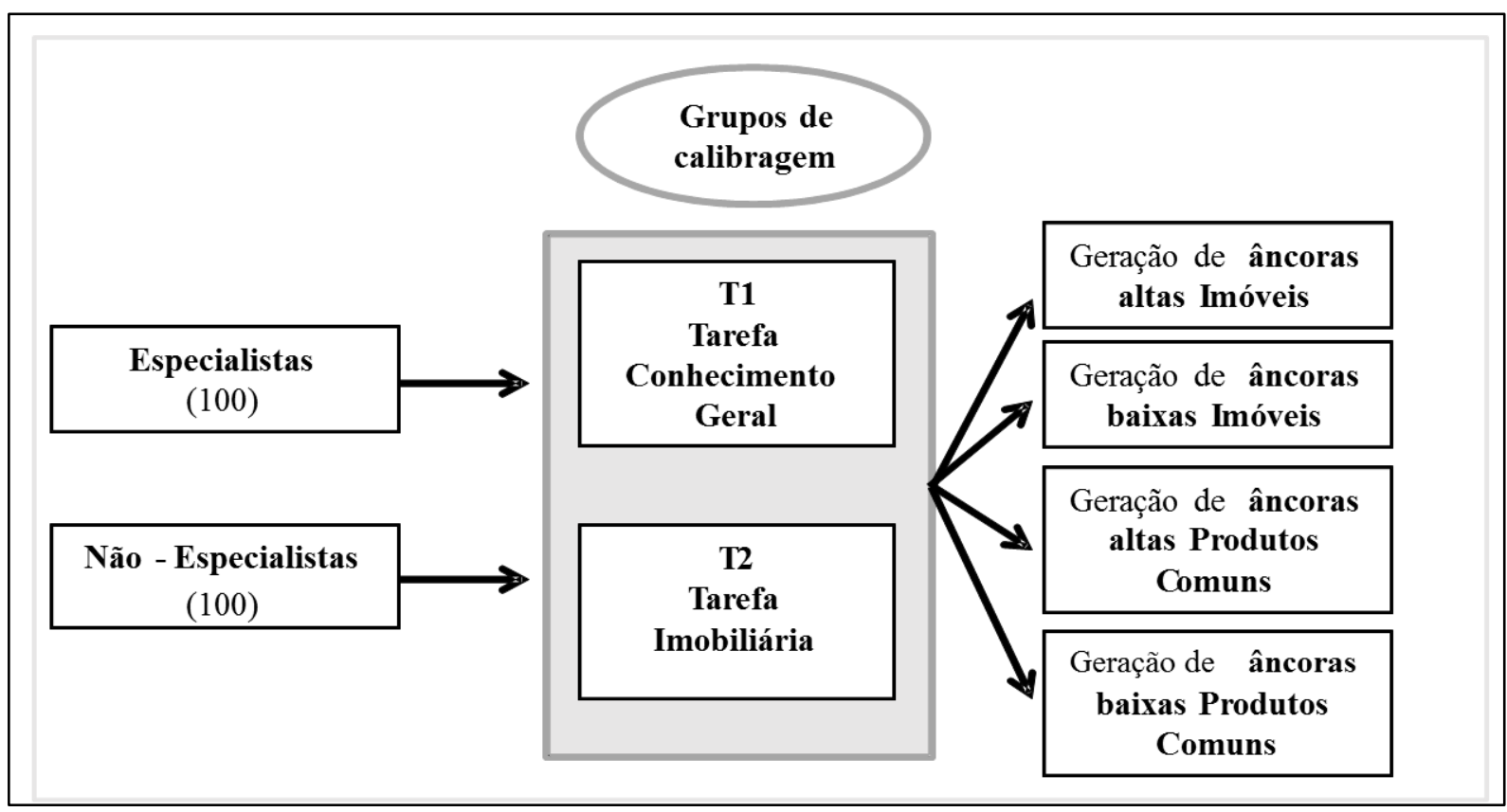

Figura 1. Desenho de Pesquisa Proposto (Fase 1)

Fonte: Elaborada pelos autores.

Tendo em vista a Figura 1, observa-se que a pesquisa utilizou duas tarefas decisórias (T1 e T2), que foram aplicadas aos sujeitos decisores (especialistas e não especialistas). A aplicação de tais tarefas decisórias seguiu o Modelo proposto por Jacowitz e Kahneman (1995) e está detalhado a seguir na página 340 .

A tarefa decisória $\mathrm{T} 1$ refere-se a produtos de conhecimento geral, contendo questões relacionadas à estimativa de preço de bens de consumo, como uma caixa de bombons, um par de óculos e um relógio. Tal tarefa decisória foi considerada nesta pesquisa como um teste de manipulação, conforme propõe Cozby (2006), pois o assunto mercado imobiliário está sendo retirado da tarefa decisória a fim de verificar se os efeitos da ancoragem se manifestam quando a variável de controle conhecimento é retirada do experimento, consistindo na principal contribuição adicional deste estudo se comparado às outras pesquisas já realizadas. Cozby (2006) afirma que o teste de manipulação consiste em uma tentativa para averiguar diretamente se a manipulação de uma variável tem o efeito pretendido sobre os respondentes. Uma tarefa decisória envolvendo bens de consumo (produtos e serviços reais) foi utilizada por Luppe (2006), que também utilizou o Modelo de Jacowitz e Kahneman (1995) para a aplicação da tarefa junto aos sujeitos decisores.

Já a tarefa decisória T2, relacionada ao mercado imobiliário, consiste em um questionário composto por questões referentes a estimativas numéricas de propriedades imobiliárias: uma casa, um apartamento e um terreno. Tarefas decisórias relacionadas ao mercado imobiliário já foram utilizadas por Northcraft e Neale (1987) e Dorow (2009).

A Figura 1 demonstra a primeira etapa do procedimento de pesquisa, com o Grupo de Calibragem, que respondeu duas questões consecutivas, fornecendo estimativas sem interferência de qualquer âncora e indicando o grau de confiança nos valores estimados. Com base nas estimativas do Grupo de 
Calibragem, foram selecionadas as âncoras, altas e baixas, de acordo com o $15^{\circ}$ e $85^{\circ}$ percentis da estimativa numérica dos valores.

Após tais definições, o Grupo Experimental fez suas estimativas, respondendo três questões consecutivas baseadas nas âncoras propostas e indicando seu grau de confiança. A Figura 2, a seguir, demonstra o processo após a realização da tarefa pelo Grupo de Calibragem, apresentando o Grupo Experimental, o qual também realizou ambas as tarefas, T1 e T2.

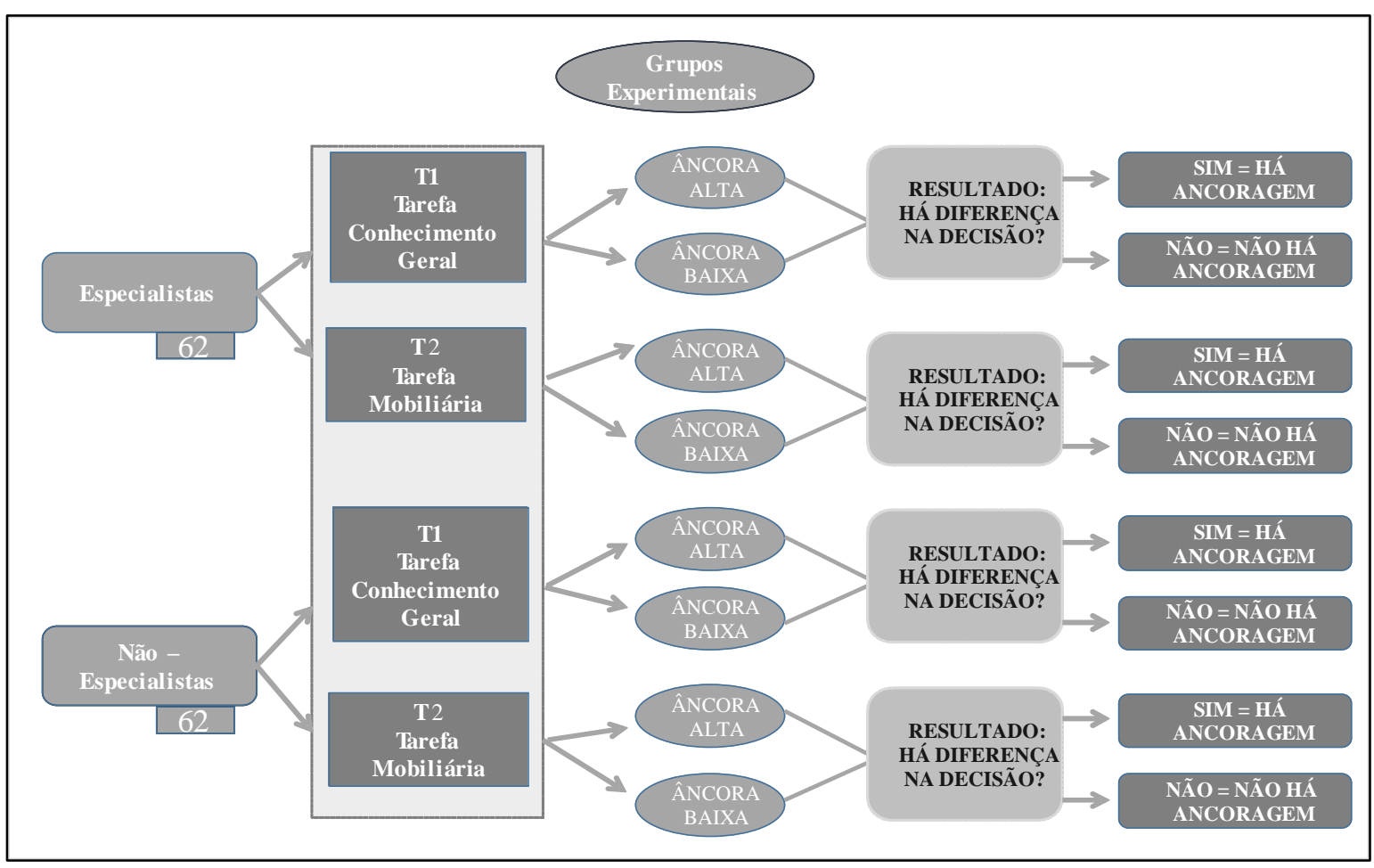

Figura 2. Desenho de Pesquisa Proposto (Fase 2)

Fonte: Elaborada pelos autores.

Nesta pesquisa, partiu-se de uma amostragem não probabilística por conveniência, pois não se fez uso de formas aleatórias de seleção das amostras. Na técnica de amostragem por conveniência, são selecionados os elementos da amostra disponíveis para participação no estudo (Hair, Anderson, Tatham, \& Black, 2005).

Assim, a amostra foi determinada de forma intencional, com base na disponibilidade e acessibilidade dos respondentes, leigos e especialistas. A intencionalidade característica da abordagem experimental é necessária quando se trabalha com grupos experimentais e pesquisas de tal natureza.

O número de participantes, especialistas e não especialistas, foi definido com base no estudo de Jacowitz e Kahneman (1995) e Dorow (2009) - ambos utilizaram 100 sujeitos decisores no Grupo de Calibragem e 62 sujeitos decisores no Grupo Experimental. Dessa forma, foram utilizados 324 sujeitos decisores nesta pesquisa: 100 especialistas, corretores de imóveis atuantes no mercado imobiliário, participaram da primeira etapa da pesquisa (Grupo de Calibragem), e 62 corretores fizeram parte da segunda etapa (Grupos Experimentais). Todos os corretores de imóveis participantes do estudo atuam junto a imobiliárias de uma única cidade e possuem registro no Conselho Regional de Corretores de Imóveis (CRECI).

Em relação aos sujeitos decisores não especialistas, os quais consistiram em alunos de final de curso de graduação, alunos de pós-graduação e servidores públicos, todos sem experiência no mercado imobiliário, foram considerados em número igual ao de especialistas, ou seja, 100 participaram da primeira etapa da pesquisa (Grupo de Calibragem), e 62 fizeram parte da segunda etapa do estudo (Grupos Experimentais). 
Muitos estudos sobre ancoragem têm sido efetuados para verificar a presença de vieses comportamentais no processo decisório durante a realização de estimativas numéricas. No entanto, este estudo, além da proposta de mensurar os efeitos da ancoragem durante o processo decisório, apresenta como principal contribuição adicional a verificação da manifestação da ancoragem quando uma variável de controle (conhecimento) é retirada da tarefa decisória.

Assim, caso o efeito da ancoragem no processo decisório seja o mesmo quando se considera uma tarefa relacionada ao campo de domínio do especialista e fora do seu campo de conhecimento, isso significa que o conhecimento sobre o assunto relacionado à tarefa decisória não interfere na existência ou inexistência de vieses cognitivos durante o processo de tomada de decisão.

\section{Aplicação das tarefas decisórias: Modelo de Jacowitz e Kahneman (1995)}

O método utilizado no experimento é o Modelo de Jacowitz e Kahneman (1995), que sugere uma técnica inovadora para estudos quantitativos sobre os efeitos da ancoragem em tarefas de estimação.

Segundo Luppe (2006), estudos de ancoragem em tarefas de estimação frequentemente utilizam o modelo tradicional de dois estágios, em que os sujeitos decisores são inicialmente questionados se um valor particular (âncora) é maior ou menor que uma quantidade incerta, devendo, então, estimar essa quantidade. No entanto, o Modelo de Jacowitz e Kahneman (1995) difere do modelo tradicional de dois estágios, pois esses autores apresentam um parâmetro para a mensuração dos efeitos da ancoragem nas tarefas de estimação, propondo um procedimento que requer três grupos retirados de uma mesma população. Tais grupos são denominados de Grupo de Calibragem e Grupos Experimentais.

O Grupo de Calibragem, respondendo a duas questões consecutivas, fornece estimativas sem a interferência de qualquer âncora e indica o grau de confiança nos valores estimados em uma escala de dez pontos, em que zero corresponde a nenhuma confiança na estimativa realizada e dez corresponde à total confiança em sua estimativa.

Com base nas estimativas do Grupo de Calibragem (fase 1), são selecionadas as âncoras, alta e baixa, de acordo com a posição na distribuição das estimativas numéricas realizadas. O Modelo de Jacowitz e Kahneman (1995) propõe que o $15^{\circ}$ e o $85^{\circ}$ percentis na distribuição das estimativas sejam fixados como âncora baixa e âncora alta, respectivamente.

Após a definição da âncora alta e da âncora baixa, os Grupos Experimentais (fase 2) fazem suas estimativas, respondendo a três questões consecutivas, baseados nas âncoras propostas (alta ou baixa), e indicam o grau de confiança nos valores estimados, considerando uma escala de dez pontos, em que zero indica que o sujeito decisor não possui nenhuma confiança na estimativa realizada e dez indica que possui total confiança em sua estimativa.

Jacowitz e Kahneman (1995), para a análise descritiva dos efeitos da ancoragem, utilizam um índice de ancoragem (IA) com o objetivo de medir o movimento da estimativa mediana dos sujeitos decisores que compõem os Grupos Experimentais em direção à âncora a que foram expostos.

\section{Técnicas de tratamento e análise de dados}

\section{Índice de ancoragem (IA) proposto por Jacowitz e Kahneman (1995)}

Com o intuito de realizar uma análise descritiva dos efeitos da heurística da ancoragem nas estimativas numéricas realizadas pelos sujeitos decisores, Jacowitz e Kahneman (1995) propõem a utilização de um índice de ancoragem (IA). Tal índice objetiva medir o movimento da estimativa mediana dos sujeitos decisores que compõem os Grupos Experimentais em direção à âncora a que foram expostos. 
Assim, foi calculado o IA para cada questão (conhecimento geral e mercado imobiliário), de acordo com as estimativas realizadas pelos sujeitos decisores integrantes dos Grupos Experimentais de especialistas e não especialistas, por meio das Equações 1, 2 e 3:

Índice de Ancoragem (IA) para um problema particular

$$
I A=\frac{\text { mediana (âncoraAlta)-mediana (âncoraBaixa) }}{\text { âncoraAlta-âncoraBaixa }}
$$

Índice de Ancoragem (IA) para âncora baixa

$$
I A=\frac{\text { mediana }(\text { âncoraBaixa })-\text { mediana }(\text { grupoCalibragem })}{\text { âncoraBaixa-mediana }(\text { grupoCalibragem })}
$$

Índice de Ancoragem (IA) para âncora alta

$$
I A=\frac{\text { mediana }(\text { âncoraAlta })-\text { mediana }(\text { grupoCalibragem })}{\text { âncoraAlta-mediana }(\text { grupoCalibragem })}
$$

O Modelo de Jacowitz e Kahneman (1995) define que a plausibilidade dos valores obtidos para o IA varia de zero (nenhum efeito da ancoragem) a um (quando a estimativa mediana dos sujeitos decisores dos Grupos Experimentais coincide com a âncora a que foram expostos). Valores superiores também podem ser encontrados.

Para valores de IA entre esse intervalo de zero e um, o referido índice é interpretado da seguinte forma: IA $=0,60$, significa que as medianas das estimativas dos sujeitos do Grupo Experimental se moveram mais que $60 \%$ em direção à âncora a que foram expostos em relação às medianas das estimativas do Grupo de Calibragem.

\section{Verificação de evidências da heurística da ancoragem nas estimativas dos grupos experimentais: teste de médias}

Os efeitos das âncoras altas e das âncoras baixas nas estimativas realizadas pelos sujeitos decisores podem ser mensurados pela comparação das médias das estimativas transformadas nos Grupos Experimentais (Luppe, 2006). Assim, para verificação das diferenças entre as estimativas com âncoras altas e baixas, foi realizado um teste estatístico $t$ para amostras independentes, como o intuito de identificar se há diferença estatisticamente significativa entre as médias das estimativas transformadas dos Grupos Experimentais.

Vieira (1980) afirma que o teste $t$ possibilita comparar médias de uma ou duas amostras, permitindo verificar se existem ou não diferenças significativas entre as médias dos grupos. Para a aplicação do teste $t$, é necessário contar com uma amostra superior a trinta e uma distribuição das variáveis próxima de uma distribuição normal (Kazmier, 1982). Ademais, para a análise da heurística da ancoragem, foi adotado um intervalo de confiança de $95 \%$, ou seja, $\alpha=5 \%$.

A distribuição de normalidade das estimativas transformadas foi testada a partir do teste de Shapiro-Wilk. Assim, tendo em vista a normalidade dos dados, foi possível usar o teste $t$ para o cálculo das médias das estimativas transformadas. $\mathrm{O}$ objetivo desse procedimento estatístico foi verificar se a média das estimativas transformadas do Grupo Experimental de especiaslitas e não especialistas submetidos a uma âncora alta seria igual à média do outro Grupo Experimental de especialistas e não especialistas submetidos a questionamentos com âncora baixa. 


\section{Análise dos Resultados}

Para a análise quantitativa, os questionários (tarefas decisórias) foram tabulados em uma planilha criada no Microsoft Excel e, posteriormente, transpostos para o software SPSS 15.0 e BioEstat 5.3.

\section{Índice de ancoragem (IA) proposto por Jacowitz e Kahneman (1995)}

\section{Índice de ancoragem: especialistas}

A Tabela 1 apresenta os índices de ancoragem obtidos a partir das estimativas numéricas de especialistas referentes a produtos de conhecimento geral e propriedades imobiliárias. Em relação aos produtos de conhecimento geral (T1), os efeitos da ancoragem são notoriamente grandes. Entre as três questões da primeira tarefa decisória, a média do IA foi de 0,70. Assim, as medianas das estimativas do Grupo Experimental composto por especialistas se moveram $70 \%$ em direção à âncora em relação às medianas das estimativas do Grupo de Calibragem.

Tabela 1

Índices de Ancoragem - Especialistas: Produtos de Conhecimento Geral e Propriedades Imobiliárias

\begin{tabular}{lcccccccc}
\hline Produto & IA Geral & $\begin{array}{c}\text { IA Âncora } \\
\text { Baixa }\end{array}$ & $\begin{array}{c}\text { IA Âncora } \\
\text { Alta }\end{array}$ & $\begin{array}{c}\text { Propriedade } \\
\text { Imobiliária }\end{array}$ & IA Geral & $\begin{array}{c}\text { IA Âncora } \\
\text { Baixa }\end{array}$ & $\begin{array}{c}\text { IA Âncora } \\
\text { Alta }\end{array}$ \\
\hline Caixa de bombons & $\mathbf{0 , 8 9}$ & $\mathbf{0 , 2 2}$ & $\mathbf{1 , 0 0}$ & Casa & $\mathbf{0 , 1 1}$ & $\mathbf{0 , 3 3}$ & $\mathbf{0 , 3 3}$ \\
\hline Óculos & $\mathbf{0 , 6 1}$ & $\mathbf{0 , 5 5}$ & $\mathbf{0 , 6 6}$ & Apartamento & $\mathbf{0 , 1 8}$ & $\mathbf{0 , 0 0}$ & $\mathbf{0 , 4 2}$ \\
\hline Relógio & $\mathbf{0 , 5 9}$ & $\mathbf{0 , 6 5}$ & $\mathbf{0 , 5 5}$ & Terreno & $\mathbf{0 , 1 4}$ & $\mathbf{0 , 3 1}$ & $\mathbf{0 , 0 0}$ \\
\hline Média & $\mathbf{0 , 7 0}$ & $\mathbf{0 , 4 7}$ & $\mathbf{0 , 9 0}$ & Média & $\mathbf{0 , 1 4}$ & $\mathbf{0 , 2 1}$ & $\mathbf{0 , 2 5}$ \\
\hline
\end{tabular}

Observa-se que as estimativas mais influenciadas pela âncora alta foram as referentes à caixa de bombons, sofrendo uma influência notória do valor inicial (IA superior a 1,0). No entanto, o IA mais baixo $(0,22)$ foi obtido para as estimativas numéricas da caixa de bombons, realizadas pelo grupo de especialistas exposto à âncora baixa.

É possível constatar, também, que, além das estimativas da caixa de bombons, as estimativas relacionadas aos óculos sofreram uma influência maior da âncora alta $(\mathrm{IA}=0,66)$. Já em relação às estimativas do relógio, a âncora baixa mostrou maior influência $(\mathrm{IA}=0,65)$ que a âncora alta.

Assim, em geral, verifica-se que a âncora alta foi o valor que mais influenciou as estimativas numéricas dos especialistas ( $\mathrm{IA}=0,90$ ), ou seja, quando expostos a uma âncora alta, as estimativas dos especialistas se moveram $90 \%$ em direção à âncora em relação às medianas das estimativas do Grupo de Calibragem.

No que tange aos índices de ancoragem dos especialistas referentes à segunda tarefa decisória (propriedades imobiliárias - T2), verifica-se que são inferiores aos índices de T1. Entre as três questões da referida tarefa decisória, a média do IA foi de 0,14 , indicando que as medianas das estimativas do Grupo Experimental composto por especialistas se moveram $14 \%$ em direção à âncora em relação às medianas das estimativas do Grupo de Calibragem.

Observa-se que foi obtido, inclusive, IA = 0,00, significando que não houve ancoragem (Jacowitz \& Kahneman, 1995) nas estimativas dos especialistas, como, por exemplo, nas estimativas dos especialistas expostos a uma âncora baixa na determinação de estimativas numéricas para o apartamento 
$(\mathrm{IA}=0,00)$ e nas estimativas realizadas pelos especialistas submetidos a uma âncora alta quando realizaram estimativas para o terreno.

Tendo em vista o exposto, verifica-se que os especialistas, quando puderam fazer uso de seu conhecimento, respondendo a tarefas decisórias relacionadas a ele (T2), obtiveram índices de ancoragens muito baixos, corroborando os estudos de Smith et al. (2013). No entanto, quando realizado o teste de manipulação, em que a variável de controle é retirada, ou seja, quando os especialistas responderam a uma tarefa decisória aleatória (T1), fora do seu campo de conhecimento, a influência das âncoras foi significativa nas suas estimativas numéricas, principalmente da âncora alta. Esse resultado difere dos resultados encontrados por Luppe (2006) e Dorow (2009), que constataram que os efeitos da âncora baixa são significativamente maiores. No entanto, corroboram os achados de Jacowitz e Kahneman (1995), em que as âncoras altas foram mais eficazes que as âncoras baixas.

Além disso, os resultados ratificam o estudo de Jacowitz e Kahaneman (1995) ao demonstrar que a heurística da ancoragem afeta a tomada de decisão, ainda que tais autores tenham evidenciado que isso ocorre com índices muito maiores (IA geral $=0,70$ ) quando um sujeito decisor responde tarefas decisórias que não conhece (fora do seu campo de domínio) e índices notoriamente baixos (IA geral = $0,14)$ quando o decisor responde tarefas decisórias relacionadas ao seu conhecimento.

\section{Índice de ancoragem: não especialistas}

A Tabela 2 apresenta os índices de ancoragem obtidos a partir das estimativas numéricas de não especialistas referentes a produtos de conhecimento geral e propriedades imobiliárias. Considerando-se os produtos de conhecimento geral (T1), observa-se que, entre as três questões da primeira tarefa decisória, a média do IA foi de 0,53. Logo, as medianas das estimativas do Grupo Experimental composto por não especialistas se moveram mais que 50\% em direção à âncora em relação às medianas das estimativas do Grupo de Calibragem.

Tabela 2

Índices de Ancoragem - Não Especialistas: Produtos de Conhecimento Geral e Propriedades Imobiliárias

\begin{tabular}{lccccccc}
\hline Produto & $\begin{array}{c}\text { IA } \\
\text { Geral }\end{array}$ & $\begin{array}{c}\text { IA Âncora } \\
\text { Baixa }\end{array}$ & $\begin{array}{c}\text { IA Âncora } \\
\text { Alta }\end{array}$ & $\begin{array}{c}\text { Propriedade } \\
\text { Imobiliária }\end{array}$ & $\begin{array}{c}\text { IA } \\
\text { Geral }\end{array}$ & $\begin{array}{c}\text { IA Âncora } \\
\text { Baixa }\end{array}$ & $\begin{array}{c}\text { IA Âncora } \\
\text { Alta }\end{array}$ \\
\hline Caixa de bombons & $\mathbf{0 , 4 0}$ & $\mathbf{0 , 2 9}$ & $\mathbf{0 , 5 7}$ & Casa & $\mathbf{0 , 5 0}$ & $\mathbf{0 , 1 5}$ & $\mathbf{0 , 8 1}$ \\
\hline Óculos & $\mathbf{0 , 5 6}$ & $\mathbf{0 , 2 8}$ & $\mathbf{0 , 8 3}$ & Apartamento & $\mathbf{0 , 7 1}$ & $\mathbf{0 , 5 5}$ & $\mathbf{0 , 7 9}$ \\
\hline Relógio & $\mathbf{0 , 6 3}$ & $\mathbf{0 , 4 2}$ & $\mathbf{0 , 7 4}$ & Terreno & $\mathbf{0 , 5 7}$ & $\mathbf{0 , 6 6}$ & $\mathbf{0 , 5 2}$ \\
\hline Média & $\mathbf{0 , 5 3}$ & $\mathbf{0 , 3 3}$ & $\mathbf{0 , 7 1}$ & Média & $\mathbf{0 , 6 0}$ & $\mathbf{0 , 4 5}$ & $\mathbf{0 , 7 1}$ \\
\hline
\end{tabular}

É possível identificar que as estimativas mais influenciadas pela âncora alta foram aquelas referentes aos óculos, sofrendo uma influência notória do valor inicial $(I A=0,83)$. No entanto, o IA mais baixo $(0,28)$ foi obtido para as estimativas numéricas dos óculos realizadas pelo grupo de não especialistas exposto à âncora baixa.

Observa-se, ainda, que, além das estimativas dos óculos, as estimativas relacionadas à caixa de bombons e ao relógio sofreram uma influência maior da âncora alta, IA $=0,57$ e IA $=0,74$, respectivamente.

Assim, em geral, verifica-se que a âncora alta foi o valor que mais influenciou as estimativas numéricas dos não especialistas em mercado imobiliário (IA $=0,70$ ), ou seja, os não especialistas, quando expostos a uma âncora alta, apresentaram estimativas que se moveram mais que $70 \%$ em direção à âncora em relação às medianas das estimativas do Grupo de Calibragem. 
Quanto aos índices de ancoragem dos sujeitos decisores não especialistas referentes à segunda tarefa decisória (propriedades imobiliárias - T2), verifica-se que são semelhantes aos índices de T1. Entre as três questões da referida tarefa decisória, a média do IA foi de 0,60 , indicando que as medianas das estimativas do Grupo Experimental composto por não especialistas se moveram $60 \%$ em direção à âncora em relação às medianas das estimativas do Grupo de Calibragem.

Observam-se, mais uma vez, índices de ancoragem maiores para estimativas dos sujeitos decisores expostos à âncora alta, contrariando os resultados encontrados por Luppe (2006) e Dorow (2009), mas confirmando os resultados encontrados por Jacowitz e Kahneman (1995).

É relevante destacar, também, o índice de ancoragem das estimativas numéricas da casa realizada por não especialistas expostos à âncora baixa, o qual demonstra que apenas $15 \%$ das estimativas se moveram em direção à âncora. Em relação aos outros índices, observa-se novamente a notória influência da âncora alta nas estimativas, apresentando IA $=0,81$ para a casa e IA $=0,79$ para o apartamento. Quanto às estimativas referentes ao terreno, é possível constatar que foram mais influenciadas pelo valor de âncora baixa.

Por fim, torna-se pertinente ressaltar que, como nenhuma das tarefas decisórias (T1 e T2) consistia em objeto de conhecimento dos sujeitos decisores não especialistas, observaram-se índices de ancoragem muito semelhantes, superiores a 0,50 .

Sob essa perspectiva, é possível verificar que o IA geral dos não especialistas referentes às três questões de conhecimento geral também foi superior a 0,50. Assim, pode-se inferir para esta pesquisa que, quando os sujeitos decisores não possuíam conhecimento a respeito da tarefa decisória, suas estimativas numéricas se moveram mais de $50 \%$ em direção à âncora a que foram expostos.

\section{Verificação de evidências da heurística da ancoragem nas estimativas dos grupos experimentais: teste de médias}

Para verificação das diferenças entre as estimativas com âncoras altas e baixas, foi realizado um teste estatístico $t$ para duas amostras independentes, com o intuito de identificar se há diferença estatisticamente significativa entre as médias das estimativas transformadas dos Grupos Experimentais. Segundo Norussis (1998), o teste $t$ para duas amostras independentes permite comparar a média da variável de um grupo com a média da mesma variável em outro grupo. Nesse sentido, a Tabela 3 apresenta os valores encontrados a partir do teste $t$ para amostras independentes, considerando-se sujeitos decisores especialistas e não especialistas em mercado de imóveis, respectivamente.

Tabela 3

Testes $\boldsymbol{t}$ para as Estimativas Transformadas: Especialistas

\begin{tabular}{llllll}
\hline \multirow{2}{*}{ Tarefa Decisória } & \multicolumn{2}{c}{ Especialistas } & \multicolumn{2}{l}{ Não-Especialistas } \\
\cline { 2 - 6 } & & Teste $\boldsymbol{t}$ & $\mathrm{Sig}$. & Teste $\boldsymbol{t}$ & Sig \\
\hline \multirow{2}{*}{ T1 } & Caixa de bombons & 7,0046 & $\mathrm{p}<0,0001$ & 4,8385 & $\mathrm{p}<0,0001$ \\
\cline { 2 - 6 } & Óculos & 7,8635 & $\mathrm{p}<0,0001$ & 5,8100 & $\mathrm{p}<0,0001$ \\
\cline { 2 - 6 } & Relógio & 5,3872 & $\mathrm{p}<0,0001$ & 7,7683 & $\mathrm{p}<0,0001$ \\
\hline \multirow{2}{*}{ T2 } & Casa & 3,2820 & $\mathrm{p}=0,0575$ & 4,6394 & $\mathrm{p}<0,0001$ \\
\cline { 2 - 6 } & Apartamento & 3,5334 & $\mathrm{p}=0,0528$ & 9,7740 & $\mathrm{p}<0,0001$ \\
\cline { 2 - 6 } & Terreno & 1,7900 & $\mathrm{p}=0,0784$ & 8,5828 & $\mathrm{p}<0,0001$ \\
\hline
\end{tabular}

A distribuição de normalidade das estimativas transformadas, como já mencionado, foi testada por meio do teste de Shapiro-Wilk. Assim, tendo em vista a normalidade dos dados, foi possível utilizar o teste $t$ para o cálculo das médias das estimativas transformadas. 
Por meio da análise da Tabela 3, observa-se que, para os sujeitos decisores não especialistas, existem diferenças de média altamente significantes $(\mathrm{p}<0,0001)$ nas três questões da primeira tarefa decisória (T1) e nas três questões da segunda tarefa decisória (T2). Logo, rejeita-se a hipótese de nulidade $\left(H_{0}: \mu_{A}=\mu_{B}\right)$ e aceita-se a hipótese alternativa $\left(H_{1}: \mu_{A} \neq \mu_{B}\right)$. Assim, com base no teste $t$, verifica-se que há evidências da heurística da ancoragem no processo decisório dos indivíduos integrantes do Grupo Experimental de não especialistas (expostos à âncora alta e âncora baixa), tanto em T1 quanto em T2.

No que tange aos especialistas, é possível perceber que existem diferenças de média altamente significantes $(\mathrm{p}<0,0001)$ para as questões integrantes da primeira tarefa decisória $(\mathrm{T} 1)$. Assim, para produtos de conhecimento geral, rejeita-se $H_{0}$ e aceita-se $H_{1}: \mu_{A} \neq \mu_{B}$. Logo, em T1 há evidências da heurística da ancoragem no processo decisório dos especilialistas. Resultados que vão ao encontro dessa constatação também foram evidenciados por Jacowitz e Kahneman (1995) e Luppe (2006), os quais identificaram que sujeitos decisores ancoram durante seu processo decisório.

No entanto, em relação à segunda tarefa decisória (T2), observa-se que o valor de $t$ não é significativo, ou seja, a diferença entre as médias das estimativas transformadas não é estatisticamente significante. Logo, aceita-se $H_{0}: \mu_{A}=\mu_{B}$ e infere-se que, em T2, não são encontradas evidências da ancoragem no processo decisório dos especialistas, contrariando os resultados encontrados por Northcraft e Neale (1987) e Dorow (2009), os quais perceberam evidências da ancoragem no processo decisório de corretores imobiliários quando submetidos à realização de estimativas referentes ao mercado de imóveis.

\section{Considerações Finais}

Desde o início dos anos 2000, vários estudos têm testado o fenômeno da ancoragem em uma ampla gama de situações (Jetter \& Walker, 2017). Pessoas comuns, investidores e corretores profissionais de imóveis, ao descobrirem que nem sempre mantêm a racionalidade plena, podem vir a diminuir a suscetibilidade das heurísticas e, como consequência, diminuir a incidência de prejuízos e/ou aumentar a possibilidade de satisfação (Northcraft \& Neale, 1987; Simon, 1991).

Nesse contexto, decisões que envolvam estimativas numéricas, como é o caso desta pesquisa, devem ser tratadas com demasiada atenção, pois, conforme Chapman e Johnson (2002), os valores numéricos (âncoras) em julgamentos e decisões afetam a performance dos decisores. As possíveis causas das falhas nos julgamentos sob influência de uma âncora, para Baghestanian e Walker (2015), provêm da intenção do decisor de simplificar o processo, influenciando a sua decisão e distorcendo julgamentos, e também do nível de incertezas e mediadores ante o processo (Zain, Farooqb, Sultanaa, \& Farooqc, 2017).

Assim, uma vez identificado o não conhecimento da heurística da ancoragem por parte de um negociante, no momento de um fechamento de negócio, a definição do preço pode ser direcionada para o objetivo da pessoa que detém o conhecimento. Se o preço do imóvel é manipulado a ponto de a pessoa que irá comprar o imóvel tomá-lo como âncora, provavelmente aquele que conhece esse viés cognitivo irá conseguir direcionar ou redirecionar o fechamento do negócio em seu favor (Reina, Dorow, Macedo, Reina, \& Nunes, 2009).

Considerando a afirmação de Mussweiler e Strack (2000), os quais declaram que a ancoragem consiste em uma das influências mais notáveis em julgamento e tomada de decisão, o presente estudo buscou verificar os efeitos da referida heurística no processo decisório de especialistas e não especialistas em mercado imobiliário. Segundo Northcraft e Neale (1987), a ancoragem tem sido demonstrada independentemente do conhecimento especializado dos respondentes no assunto proposto. No entanto, Thorsteinson et al. (2008) argumentam que os efeitos da ancoragem são reduzidos quando 
sujeitos decisores têm mais conhecimento acerca dos problemas em questão. Da mesma forma, Welsh et al. (2014) defendem que, se há confiança de que a pessoa realizando uma estimativa consiste em um especialista, é plausível esperar que suas estimativas sejam menos afetadas pelos efeitos da ancoragem.

Diante disso, o objetivo principal desta pesquisa foi verificar a existência da heurística da ancoragem nas estimativas de especialistas e não especialistas em mercado imobiliário, quando submetidos a tarefas experimentais relacionadas ao campo de domínio do especialista e fora do seu campo de conhecimento. Tal objetivo foi alcançado por meio da realização de um experimento, mediante a aplicação de duas tarefas decisórias (T1 e T2) junto a Grupos de Calibragens e Grupos Experimentais compostos por especialistas e não especialistas em mercado imobiliário, utilizando o Modelo de Jacowitz e Kahneman (1995). Esse modelo difere do modelo tradicional de dois estágios, tradicionalmente usado, pois os referidos autores apresentam um parâmetro para a mensuração dos efeitos da ancoragem nas tarefas de estimação e propõem um procedimento para medir a ancoragem que requer três grupos retirados de uma mesma população, denominados de Grupo de Calibragem e Grupos Experimentais.

Os resultados que contribuíram para o alcance do objetivo principal demonstraram, por meio do teste estatístico $t$ para amostras independentes, que há evidências da heurística da ancoragem no processo decisório dos indivíduos integrantes do Grupo Experimental de não especialistas (âncora baixa e âncora alta), tanto em T1 quanto em T2. No que tange aos especialistas, foi possível perceber que, em T1, há evidências da heurística da ancoragem, corroborando os resultados encontrados por Jacowitz e Kahaneman (1995) e Luppe (2006). No entanto, em relação à segunda tarefa decisória (T2), observouse que as diferenças de médias não foram estatisticamente significantes, inferindo-se que, em T2, não são encontradas evidências da ancoragem no processo decisório dos especialistas, contrariando os resultados demonstrados por Northcraft e Neale (1987) e Dorow (2009), os quais encontraram evidências da ancoragem no processo decisório de corretores imobiliários quando submetidos à realização de estimativas referentes ao mercado de imóveis.

Assim, considerando o exposto, apresenta-se novamente o desenho de pesquisa proposto neste estudo (Figura 2), porém, neste momento, com os resultados encontrados.

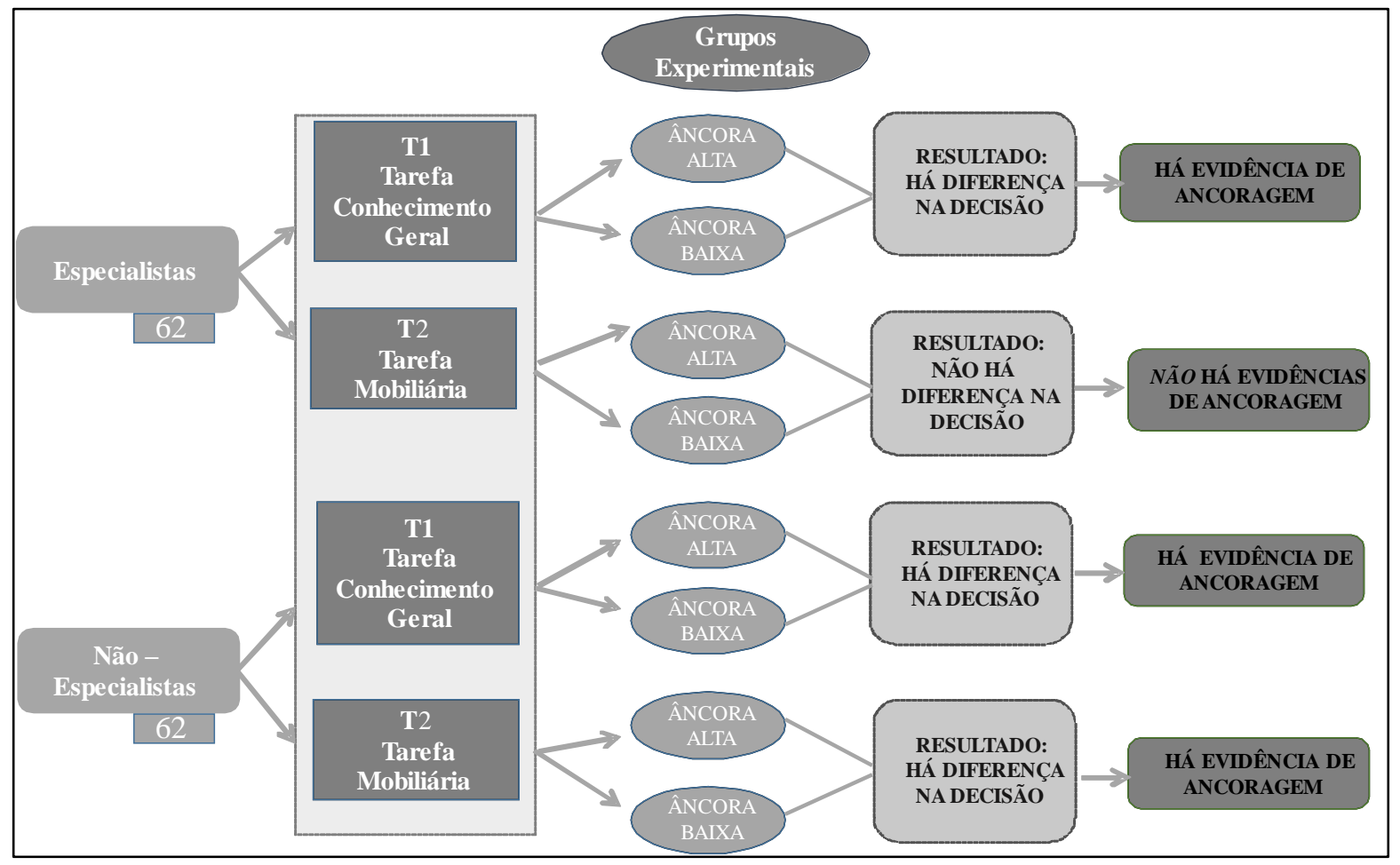

Figura 3. Desenho de Pesquisa com os Resultados Encontrados Fonte: Elaborada pelos autores. 
Em relação ao grau de ancoragem (índice) das estimativas numéricas realizadas por especialistas e não especialistas, pretendeu-se identificar se os efeitos da ancoragem são reduzidos quando sujeitos decisores têm mais conhecimento acerca dos problemas em questão. Assim, tendo em vista o Modelo proposto de Jacowitz e Kahneman (1995), foi encontrado um índice de ancoragem geral para os especialistas em mercado de imóveis igual a 0,14 quando a tarefa decisória era relacionada ao campo de domínio do especialista e um IA $=0,70$ quando a tarefa decisória estava fora do seu campo de conhecimento, ou seja, quando o sujeito decisor conhecia o assunto, suas estimativas foram deslocadas $14 \%$ em direção à âncora. No entanto, quando foi retirada a variável conhecimento do experimento, as estimativas dos especialistas se moveram $70 \%$ em direção à âncora.

Adicionalmente ao que foi discutido até aqui, é importante ressaltar que muitos estudos sobre ancoragem têm sido realizados a fim de verificar a presença da heurística da ancoragem no processo de julgamento e tomada de decisão. Para Turner e Schley (2016), a abundância de pesquisas de ancoragem evidencia sua prevalência e estabilidade como uma característica do julgamento e da tomada de decisão do ser humano.

No entanto, este estudo, além da proposta de averiguar os efeitos da ancoragem durante o processo decisório relacionado a decisões quantitativas, apresentou como principal contribuição adicional a verificação da manifestação da ancoragem quando uma variável de controle (conhecimento) é retirada da tarefa decisória. Tal procedimento é denominado por Cozby (2006) de teste de manipulação, consistindo em uma tentativa de medir diretamente se a manipulação de uma variável tem o efeito pretendido sobre os respondentes.

Assim, quando o sujeito decisor especialista foi submetido a uma tarefa decisória relacionada ao seu campo de conhecimento, não foram verificadas evidências da ancoragem em seu processo de tomada de decisão. No entanto, quando submetidos a tarefas decisórias aleatórias, não relacionadas a seu campo de domínio, constatou-se que os sujeitos decisores apresentaram evidências da ancoragem na realização de estimativas numéricas durante a aplicação das tarefas decisórias.

Sob essa perspectiva, sugere-se que estudos futuros busquem explorar o Modelo de Jacowitz e Kahneman (1995) por meio da aplicação de tarefas decisórias junto a outros profissionais especialistas, não só realizando comparações entre diferentes níveis de conhecimento dos sujeitos decisores, mas, também, considerando tarefas decisórias contendo variáveis que possam ser controladas e manipuladas para verificação dos consequentes efeitos.

\section{Referências}

Abdin, S. Z. ul, Farooq, O., Sultana, N., \& Farooq, M. (2017). The impact of heuristics on investment decision and performance: Exploring multiple mediation mechanisms. Research in International Business and Finance, 42, 674-688. http://doi:10.1016/j.ribaf.2017.07.010

Bazerman, M. H. (2004). Processo decisório: Para cursos de administração e economia (8a ed.). Rio de Janeiro: Elsevier.

Baghestanian, S., \& Walker, T. B. (2015). Anchoring in experimental asset markets. Journal of Economic Behavior and Organization, 116, 15-25. http://doi.org/10.1016/j.jebo.2015.03.010

Campbell, D. T., \& Stanley, J. C. (1979). Delineamentos experimentais e quase-experimentais de pesquisa. São Paulo: EPU/EDUSP.

Ceschi, A., Costantini, A., Sartori, R., Weller, J., \& Di Fabio, A. (in press). Dimensions of decisionmaking: An evidence-based classification of heuristics and biases. Personality and Individual Differences. http://doi.org/10.1016/j.paid.2018.07.033 
Chao, Y.-C. (2011). Decision-making biases in the alliance life cycle. Management Decision, 49(3), 350-364. https://doi.org/10.1108/00251741111120743

Chapman, G. B., \& Johnson, E. J. (2002). Incorporating the irrelevant: anchors in judgements of belief and value. In T. D. G. Gilovich \& D. Kahneman (Orgs.), Heuristics and biases: the psychology of intuitive thought (pp. 120-138). New York: Cambridge University Press.

Costa, D. F. (2017). Ensaios sobre vieses cognitivos no processo de tomada de decisão gerencial (Tese de doutorado). Universidade Federal de Lavras, Lavras, MG, Brasil.

Cozby, P. C. (2006). Métodos de pesquisa em ciências do comportamento (7a ed.). São Paulo: Atlas.

Dorow, A. (2009). Heurística da ancoragem na estimativa de preços de imóveis por corretores profissionais (Dissertação de mestrado). Universidade Federal de Santa Catarina, Florianópolis, $\mathrm{SC}$, Brasil.

Furnham, A., \& Boo, H. C. (2011). A literature review of the anchoring effect. The Journal of SocioEconomics, 40(1), 35-42. https://doi.org/10.1016/j.socec.2010.10.008

George, J. M., \& Dane, E. (2016). Affect, emotion, and decision making. Organizational Behavior and Human Decision Processes, 136, 47-55. https://doi.org/10.1016/j.obhdp.2016.06.004

Gergaud, O., Plantinga, A. J., \& Ringeval-Deluze, A. (2017). Anchored in the past: Persistent price effects of obsolete vineyard ratings in France. Journal of Economic Behavior and Organization, 133, 39-51. https://doi.org/10.1016/j.jebo.2016.10.005

Hair, J. F., Jr., Anderson, R. E., Tatham, R. L., \& Black, W. C. (2005). Análise multivariada de dados (5a ed.). Porto Alegre: Bookman.

Jacowitz, K. E., \& Kahneman, D. (1995). Measures of anchoring in estimation tasks. Personality and Social Psychology Bulletin, 21(11), 1161-1166. https://doi.org/10.1177/01461672952111004

Jetter, M., \& Walker, J. K. (2017). Anchoring in financial decision-making: Evidence from jeopardy! Journal of Economic Behavior and Organization, 141, 164-176. http://doi.org/10.1016/j.jebo.2017.07.006

Jung, H., \& Young, M. J. (2012). The de-biasing effect of incidental anger on other provided anchors. Journal of Behavioral Decision Making, 25(5), 435-442. https://doi.org/10.1002/bdm.739

Kazmier, L. J. (1982). Estatística aplicada à economia e administração. São Paulo: McGraw-Hill.

Löbler, M. L., Reis, E., Nishi, J. M., \& Tagliapietra, R. D. (2015, setembro). Inventário de estilos de tomada de decisão: Validação de instrumento no contexto brasileiro. Anais do Encontro Nacional da Associação Nacional de Pós-Graduação e Pesquisa em Administração, Belo Horizonte, MG, Brasil, 39.

Loschelder, D. D., Friese, M., \& Trötschel, R. (2017). How and why precise anchors distinctly affect anchor recipients and senders. Journal of Experimental Social Psychology, 70, 164-176. http://doi.org/10.1016/j.jesp.2016.11.001

Luppe, M. R. (2006). A heurística da ancoragem e seus efeitos no julgamento: Decisões de consumo (Dissertação de mestrado). Universidade de São Paulo, São Paulo, SP, Brasil.

Malhotra, N. K. (2006). Pesquisa de marketing: Uma orientação aplicada (4a ed.). Porto Alegre: Bookman.

Malhotra, S., Zhu, P., \& Reus, T. H. (2015). Anchoring on the acquisition premium decisions of others. Strategic Management Journal 36(12), 1866-1876. https://doi.org/10.1002/smj.2314 
Magalhães, S. (2013). Racionalidade limitada na tomada de decisão o efeito de ancoragem na avaliação de curriculum vitae: Um estudo experimental (Dissertação de mestrado). Universidade do Porto, Porto, Portugal.

Meub, L., \& Proeger, T. E. (2015). Anchoring in social context. Journal of Behavioral and Experimental Economics, 55, 29-39. https://doi.org/10.1016/j.socec.2015.01.004

Mochon, D., \& Frederick, S. (2013). Anchoring in sequential judgments. Organizational Behavior and Human Decision Processes, 122(1), 69-79. http://doi.org/10.1016/j.obhdp.2013.04.002

Mussweiler, T., \& Strack, F. (2000). Numeric judgments under uncertainty: The role of knowledge in anchoring. Journal of Experimental Social Psychology, 36(5), 495-518. http://dx.doi.org/10.1006/jesp.1999.1414

Northcraft, G. B., \& Neale, M. A. (1987). Experts, amateurs, and real estate: An anchoring-and adjustment perspective on property pricing decisions. Organizational Behavior and Human Decision Processes, 39(1), 84-97. http://doi.org/10.1016/0749-5978(87)90046-X

Norussis, M. J. (1998). SPSS 8.0 guide to data analysis. New Jersey: Prentice Hall.

Rader, C. A., Soll, J. B., \& Larrick, R. P. (2015). Pushing away from representative advice: advice taking, anchoring, and adjustment. Organizational Behavior and Human Decision Processes, 130, 26-43. http://doi.org/10.1016/j.obhdp.2015.05.004

Reina, D., Dorow, A., Macedo, J. S. M., Jr., Reina, D. R. M., \& Nunes, P. (2009). Behavioral finance: Um estudo sobre a correlação entre a heurística da ancoragem e a tomada de decisão sob risco em investimentos. Revista de Informação Contábil, 3(2), 83-98.

Simon, H. A. (1955). A behavioral model of rational choice. Quarterly Journal of Economics, 69(1), 99-118. http://doi.org/10.2307/1884852

Simon, H. A. (1991). Satisficing. In J. Eatwell, M. Milgate, \& P. Neumann (Orgs.), The new palgrave: A dictionary of economics (Vol. 4, pp. 243-234). Londres: The Macmillan Press.

Smith, A. R., Windschitl, P. D., \& Bruchmann, K. (2013). Knowledge matters: Anchoring effects are moderated by knowledge level. European Journal of Social Psychology, 43(1), 97-108. http://doi.org/10.1002/ejsp.1921

Thorsteinson, T. J., Breier, J., Atwell, A., Hamilton, C., \& Privette, M. (2008). Anchoring effects on performance judgments. Organizational Behavior and Human Decision Processes, 107(1), 2940. https://doi.org/10.1016/j.obhdp.2008.01.003

Turner, B. M., \& Schley, D. R. (2016). The anchor integration model: A descriptive model of anchoring effects. Cognitive Psychology, 90, 1-47. http://doi.org/10.1016/j.cogpsych.2016.07.003

Tversky, A., \& Kahneman, D. (1974). Judgment under uncertainty: Heuristics and biases. Science, 185(4157), 1124-1131. https://doi.org/10.1126/science.185.4157.1124

Vieira, S. (1980). Introdução à bioestatística. Rio de Janeiro: Elsevier.

Von Neumann, J., \& Morgenstern, O. (1947). Theory of games and economic behavior (2a ed.). Princeton: Princeton University Press.

Welsh, M. B., Delfabbro P. H., Burns, N. R, \& Begg, S. H. (2014). Individual differences in anchoring: Traits and experience. Learning and Individual Differences, 29, 131-140. https://doi.org/10.1016/j.lindif.2013.01.002 
Zain, S., Farooqb, O., Sultanaa, N., \& Farooqc, M. (2017). The impact of heuristics on investment decision and performance: Exploring multiple mediation mechanisms. Research in International Business and Finance, 42, 674-688. http://doi.org/10.1016/j.ribaf.2017.07.010

\section{Autores}

Paula Borges Tronco

Av. Roraima, 1000, 97105-900, Santa Maria, RS, Brasil

E-mail: pb.tronco@hotmail.com

Mauri Leodir Löbler

Av. Roraima, 1000, 97105-900, Santa Maria, RS, Brasil

E-mail: mllobler@gmail.com

Leticia Gomes dos Santos

Av. Roraima, 1000, 97105-900, Santa Maria, RS, Brasil

E-mail: letig.santos@gmail.com

Juliana Mayumi Nishi

Av. Roraima, 1000, 97105-900, Santa Maria, RS, Brasil

E-mail: ju_mn5@hotmail.com

\section{Contribuições}

$1^{\circ}$ autor: Definição da pesquisa, desenvolvimento teórico e metodológico, coleta de dados e análises de resultados, escrita do texto.

$2^{\circ}$ autor: Definição da pesquisa, desenvolvimento teórico e metodológico, revisão crítica do manuscrito.

$3^{\circ}$ autor: Desenvolvimento teórico, escrita e atualização do texto, revisão e submissão.

$4^{\circ}$ autor: Definição da pesquisa, desenvolvimento teórico e metodológico, coleta de dados.

\section{Financiamento}

CAPES e CNPq, com processo de número 447249/2014-2.

\section{Conflito de Interesses}

Os autores informaram que não há conflito de interesses.

\section{Verificação de Plágio}

A RAC mantém a prática de submeter todos os documentos aprovados para publicação à verificação de plágio, mediante o emprego de ferramentas específicas, e.g.: iThenticate.

\section{Material Suplementar}

Os autores do presente artigo decidem não disponibilizar o banco de dados da pesquisa no presente momento devido à perspectiva de utilização para extração de outras relações, o que deverá resultar em novo artigo. Assim, estamos à disposição para quem interessar possa que entre em contato com quaisquer um dos autores e receba todos os esclarecimentos possíveis sobre a pesquisa. 